\title{
The prognostic value of preoperative fibrinogen-to-prealbumin ratio and a novel FFC score in patients with resectable gastric cancer
}

Shuli Tang ${ }^{1}$, Lin Lin ${ }^{1}$, Jianan Cheng ${ }^{1}$, Juan Zhao ${ }^{1}$, Qijia Xuan ${ }^{1}$, Jiayue Shao ${ }^{1}$, Yang Zhou ${ }^{2 *}$ and Yanqiao Zhang ${ }^{1 *}$

\begin{abstract}
Background: Chronic inflammation is considered as a hallmark of gastric cancer (GC) and plays a critical role in GC progression and metastasis. This study aimed to explore the prognostic values of preoperative fibrinogen-toprealbumin ratio (FPR), fibrinogen-to-albumin ratio (FAR), and novel FPR-FAR-CEA (FFC) score in patients with GC undergoing gastrectomy.

Methods: A total of 273 patients with resectable GC were included in this retrospective study. We performed Kaplan-Meier and Cox regression analyses to assess the prognostic role of preoperative FPR, FAR, and FFC score in patients with GC and analyze their relationships with clinicopathological features.

Results: Receiver operating characteristic curve (ROC) analysis revealed that the optimal cutoff values for FPR and FAR were 0.0145 and 0.0784 , respectively. The FFC score had a higher area under the ROC curve than FAR and CEA. Elevated FPR $(\geq 0.0145)$ and FAR $(\geq 0.0784)$ were significantly associated with old age, large tumor size, tumor invasion depth, lymph nodes metastasis, advanced TNM stage, large Borrmann type, and anemia status. KaplanMeier analysis showed that high FPR, FAR, and FFC score were related to poor survival. Multivariate analyses indicated that FPR, FFC score, TNM stage, and tumor size were significant independent factors for survival.
\end{abstract}

Conclusions: Preoperative FPR and FFC score could be used as prospective noninvasive prognostic biomarkers for resectable GC.

Keywords: Gastric cancer, Inflammation, Fibrinogen-to-albumin ratio, Fibrinogen-to-prealbumin ratio, FFC score, Prognostic factor

\footnotetext{
* Correspondence: yanqiaozhang@ems.hrbmu.edu.cn;

Zhouyang094@126.com

${ }^{2}$ Department of Radiology, Harbin Medical University Cancer Hospital, No.

150, Haping Road, Nangang District, Harbin 150001, China

${ }^{1}$ Department of Medical Oncology, Harbin Medical University Cancer

Hospital, No. 150, Haping Road, Nangang District, Harbin 150001, China
}

(c) The Author(s). 2020 Open Access This article is licensed under a Creative Commons Attribution 4.0 International License, which permits use, sharing, adaptation, distribution and reproduction in any medium or format, as long as you give appropriate credit to the original author(s) and the source, provide a link to the Creative Commons licence, and indicate if changes were made. The images or other third party material in this article are included in the article's Creative Commons licence, unless indicated otherwise in a credit line to the material. If material is not included in the article's Creative Commons licence and your intended use is not permitted by statutory regulation or exceeds the permitted use, you will need to obtain permission directly from the copyright holder. To view a copy of this licence, visit http://creativecommons.org/licenses/by/4.0/ The Creative Commons Public Domain Dedication waiver (http://creativecommons.org/publicdomain/zero/1.0/) applies to the data made available in this article, unless otherwise stated in a credit line to the data. 


\section{Background}

Gastric cancer (GC) is an important healthcare challenge worldwide, especially in eastern Asia. Recent estimates have indicated that GC is the fifth most common cancer and third leading cause of cancer-related mortality worldwide, with 1,000,000 newly diagnosed cases and 783,000 deaths annually [1]. In China, GC is the second leading cause of cancer mortality [2]. Despite new and available diagnostic and therapeutic strategies developed over the past several decades, prognosis for patients with GC remains poor with an overall 5-year relative survival rate of about 20\% [3]. Although TNM classification has been clinically recognized as a strong biomarker for predicting the prognosis of GC, high heterogeneity status leads to different outcomes among patients with GC even with the same TNM stage and treatments. Thus, novel effective and noninvasive prognostic biomarkers should be identified to provide information for personalized treatment and improve patient's outcomes.

Increasing evidence suggests that systemic inflammatory plays a critical role in cancer progression and metastasis, thereby demanding individualized immune-related therapeutic strategies [3, 4]. Recently, extensive attention has been given to systemic inflammation-based biomarkers in various malignancies. To date, several inflammation-based biomarkers, such as C-reactive protein, albumin, neutrophil-to-lymphocyte ratio (NLR), platelet-tolymphocyte ratio (PLR), lymphocyte-to-monocyte ratio (LMR), and Glasgow prognostic score (GPS), have been revealed as useful prognostic biomarkers in $\mathrm{GC}$, colorectal cancer $(\mathrm{CRC})$, breast cancer, and lung cancer [5-7]. In addition, high levels of fibrinogen-to-albumin ratio (FAR), a novel inflammation-based biomarker, are associated with poor outcomes in various cancers, including esophageal squamous cell carcinoma (ESCC), breast cancer, gallbladder cancer, and soft tissue sarcoma [8, 9]. More recently, the fibrinogen-to-prealbumin ratio (FPR) was demonstrated to be an excellent diagnostic and prognostic biomarker for patients with CRC and a tool to identify individuals who can benefit from adjuvant chemotherapy treatment [10]. However, the prognostic values of FPR and FAR in GC remain unclear. Thus, in this study, we comprehensively explored the prognostic values of serumbased preoperative FPR and FAR in GC and analyzed the associations between these biomarkers and clinicopathological features. Moreover, we established a novel FPRFAR-CEA (FFC) score based on inflammation as an independent predictor for patients with resectable GC.

\section{Methods}

\section{Patient population}

A total of 273 patients with resectable GC between December 2009 and December 2011 at Harbin Medical University Cancer Hospital were assessed in our study.
Patients included in the study met the following criteria: (1) patients with newly histologically confirmed gastric noncardia adenocarcinoma; (2) had no distant metastatic lesions before surgery; (3) had no evidence of other malignancies; (4) had no history of autoimmune disorders, hepatitis, HIV infection, or other recent infection; (5) received curative resection; (6) had not undergone neoadjuvant chemotherapy before surgery; and (7) had complete medical records and available follow-up data. All eligible patients gave written informed consent, and our study was approved by the Institutional Ethics Committee of Harbin Medical University Cancer Hospital.

\section{Clinical data collection and follow-up investigation}

Baseline data, including gender, age, tumor size, pathological differentiation, Borrmann type, body mass index (BMI), anemia status, and preoperative laboratory data (CEA and CA199 levels, serum fibrinogen, prealbumin, and albumin values) were gathered from medical records. All laboratory data were obtained from routine blood testing within $48 \mathrm{~h}$ after the first hospitalization. The pathological tumor stage (pTNM), tumor invasion depth, and lymph nodes metastasis of gastric cancers were identified according to the 7th edition AJCC TNM classification. Follow up of postoperative patients with GC was performed regularly until December 31, 2016 (more than 5 years) or until death. Patients with followup periods that were less than 30 days were excluded from the analysis. Overall survival (OS) was calculated from the time of operation to death or last follow up.

\section{Definition of inflammation-based indicators and a novel prognostic score}

FPR was calculated as the serum fibrinogen value divided by the serum prealbumin value. FAR was defined as the serum fibrinogen value divided by the serum albumin value. FFC score, a novel inflammation-associated prognostic score, was established to further investigate the prognostic values of FPR, FAR, and CEA in our study. The FFC score was defined as the combination of elevated FPR, FAR, and CEA; scores of $0,1,2$, or 3 were allocated based on the number of elevated levels. According to the optimal cutoff values for FPR and FAR were 0.0145 and 0.0784 , respectively. Higher or lower levels of FPR or FAR than cutoff values were scored with 1 or 0 , respectively. Patients with elevated CEA (> $5 \mathrm{ng}$ / $\mathrm{ml})$ or decreased CEA $(\leq 5 \mathrm{ng} / \mathrm{ml})$ were considered 1or 0 point, respectively.

\section{Statistical analysis}

Receiver operating characteristic (ROC) curve analysis was performed to determine the optimal cut-off values of FAR and FPR based on maximum Youden index. The area under the receiver operating characteristic curve 
(AUC) was calculated to evaluate survival prediction. The differences between the AUC values were compared using DeLong test. Permutation test was performed to validate FFC score and avoid overfitting. Chi-square $\left(\mathrm{x}^{2}\right)$ test or Fisher's exact test was performed to analyze the associations between different FPR, FAR and CEA groups and clinicopathological features. The differences among groups were estimated by Mann-Whitney $U$ test or Kruskal-Wallis test. Survival curves were evaluated and compared with each other using the Kaplan-Meier method and log-rank test to characterize the associations between inflammation-based indicators and OS. The Cox proportional hazards model was employed for univariate and multivariate analyses to assess the independent prognostic predictor for OS. Two-sided $p<0.05$ was considered statistically significant. All the statistical analyses were performed using SPSS version 20.0 (IBM Corp., Armonk, NY, USA) software and R project version 3.6.1.

\section{Results}

Baseline characteristics of patients with GC

A total of 273 patients with GC, ranging from 31 years old to 82 years old (median age of 59 years), were enrolled in the study on the basis of the inclusion criteria. All demographic and clinicopathological characteristics of patients with resectable GC are shown in Table 1 and Additional file 1: Table S1. Majority of patients were male $(72.2 \%)$ and had a poorly differentiated tumor (70.0\%). Over half of the patients (59.0\%) had tumor size less than $5 \mathrm{~cm}$, and $59.3 \%$ had lymph node metastases. A total of $89(32.6 \%)$ and $184(67.4 \%)$ patients were confirmed as $\mathrm{T} 1+2$ and $\mathrm{T} 3+4$ depth, respectively. According to the 8th edition of AJCC/UICC TNM classification, 66 (24.2\%), 78 (28.6\%), and 129 (47.2\%) were diagnosed as TNM stages I, II, and III, respectively. Adjuvant chemotherapy was performed in 172 patients. A total of $86(31.5 \%)$ patients had BMI less than 24, and $144(52.7 \%)$ patients died during the follow-up period. The median OS was 53.87 months. On the basis of Borrmann type, the number of patients with 0-IV GC types were 46 (16.8\%), 39 (14.3\%), 37 (13.6\%), 130 (47.6\%), and 21 (7.7\%), respectively. Fifty-two (19.0\%) patients had elevated CEA level, 47 (17.2\%) patients had elevated CA199 level, and 77 (28.2\%) patients developed anemia during the study.

\section{Optimal cutoff values for FPR and FAR}

As shown in Table 1, the median value of preoperative FPR was 0.0134 (range: 0.0038-0.1258), and the median value of preoperative FAR was 0.0760 (range: 0.02610.2989). The ROC curve and AUC for OS were computed to determine the optimal cutoff values for FPR and FAR (Fig. 1). According to the ROC curve for 5-year
Table 1 Baseline characteristics of gastric cancer patients

\begin{tabular}{|c|c|c|}
\hline Characteristic & Cases & Percentage (\%) \\
\hline \multicolumn{3}{|l|}{ Gender } \\
\hline Male & 197 & 72.2 \\
\hline Female & 76 & 27.8 \\
\hline \multicolumn{3}{|l|}{ Age (years) } \\
\hline$<60$ & 152 & 55.7 \\
\hline$\geq 60$ & 121 & 44.3 \\
\hline \multicolumn{3}{|l|}{ Tumor size $(\mathrm{cm})$} \\
\hline$<5$ & 161 & 59.0 \\
\hline$\geq 5$ & 112 & 41.0 \\
\hline \multicolumn{3}{|c|}{ Pathological differentiation } \\
\hline Moderate+Well & 82 & 30.0 \\
\hline Poor & 191 & 70.0 \\
\hline \multicolumn{3}{|l|}{ Invasion depth } \\
\hline $\mathrm{T} 1+\mathrm{T} 2$ & 89 & 32.6 \\
\hline $\mathrm{T} 3+\mathrm{T} 4$ & 184 & 67.4 \\
\hline \multicolumn{3}{|c|}{ Lymph nodes metastasis } \\
\hline Negative & 111 & 40.7 \\
\hline Positive & 162 & 59.3 \\
\hline \multicolumn{3}{|l|}{ TNM stage } \\
\hline I & 66 & 24.2 \\
\hline$\|$ & 78 & 28.6 \\
\hline III & 129 & 47.2 \\
\hline \multicolumn{3}{|l|}{ CEA (ng/ml) } \\
\hline$\leq 5$ & 221 & 81.0 \\
\hline$>5$ & 52 & 19.0 \\
\hline \multicolumn{3}{|l|}{ CA199 (U/ml) } \\
\hline$\leq 37$ & 226 & 82.8 \\
\hline$>37$ & 47 & 17.2 \\
\hline FPR median (range) & \multicolumn{2}{|c|}{$0.0134(0.0038-0.1258)$} \\
\hline FAR median (range) & \multicolumn{2}{|c|}{$0.0760(0.0261-0.2989)$} \\
\hline
\end{tabular}

CEA carcinoembryonic antigen; CA199 carbohydrate antigen 199; OS overall survival; $F P R$ fibrinogen-to-prealbumin ratio; $F A R$ fibrinogen-to-albumin ratio

OS (Fig. 1a, Additional file 2: Table S2), the optimal cutoff values for FPR and FAR were 0.0145 (AUC $=0.673$, $p<0.001,95 \%$ confidence interval [CI]: 0.609-0.737, sensitivity $=56.3 \%, \quad$ specificity $=77.5 \%$ ), and 0.0784 (AUC $=0.664, p<0.001,95 \%$ CI: $0.600-0.728$, sensitivity $=61.1 \%$, specificity $=70.5 \%$ ), respectively. The AUC values of FFC score and CEA were $0.710(p<0.001$, 95\% CI: $0.648-0.771)$ and $0.618(p=0.001,95 \%$ CI: $0.552-0.685)$, respectively. Moreover, FFC score was confirmed to be no overfitting by permutation test (Additional file 3: Figure S1). The comparisons of AUC values using Delong test revealed that there were statistical differences between either FAR $(p=0.025)$ or CEA $(p=$ 0.019 ) and FFC score. Nonetheless, there was no 

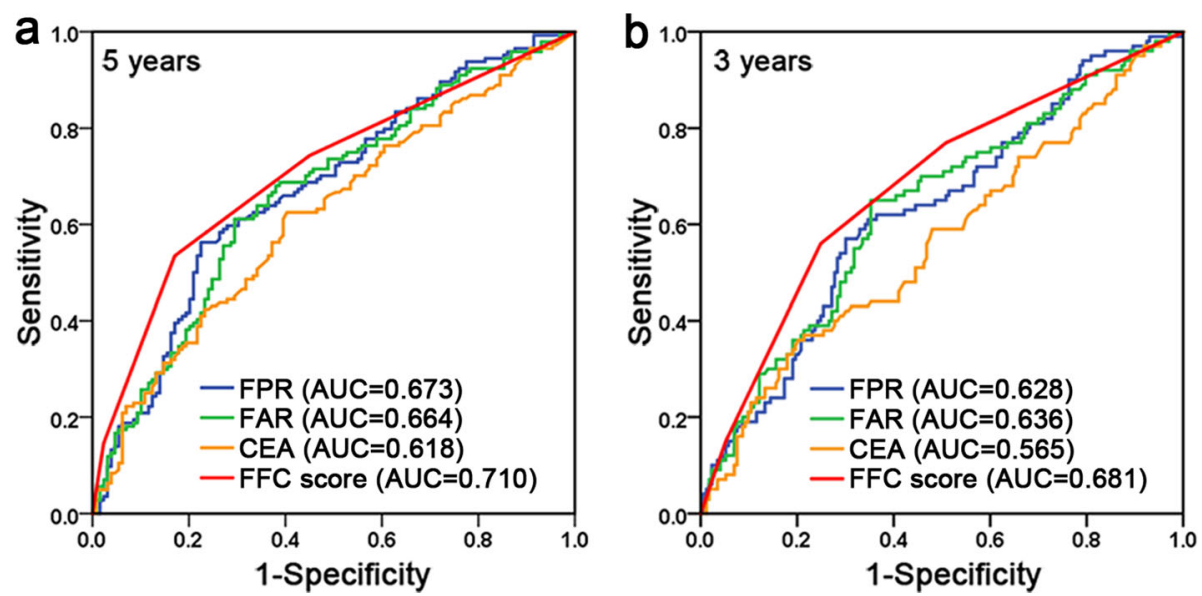

Fig. 1 ROC curve analyses of the FPR, FAR, CEA and FFC score for 5-year (a) and 3-year (b) OS in gastric cancer. Abbreviations: ROC, receiver operating characteristic; AUC, area under ROC curve; OS, overall survival; FPR, fibrinogen-to-prealbumin ratio; FAR, fibrinogen-to-albumin ratio; CEA, carcinoembryonic antigen; FFC, FPR-FAR-CEA

difference between FFC score and FPR $(p=0.125)$. In addition, as shown in Fig. 1b, the AUC values of the ROC curves for 3-year OS of FPR, FAR, CEA and FFC score were 0.628 ( $p<0.001,95 \%$ CI: 0.560-0.697), 0.636 $(p<0.001,95 \%$ CI: 0.567-0.704), 0.565 ( $p=0.075,95 \%$ CI: $0.493-0.637)$, and $0.681(p<0.001,95 \%$ CI: $0.615-$ $0.748)$, respectively. The Delong test revealed that there were statistical differences in the AUC values between either FPR $(p=0.012)$, FAR $(p=0.029)$ or CEA $(p=$ 0.003 ) and FFC score.

\section{Correlations of preoperative FPR and FAR with clinicopathological features in GC}

On the basis of the cutoff values, the patients were divided into low $(<0.0145)$ or high $(\geq 0.0145)$ FPR groups, low $(<0.0784)$ or high $(\geq 0.0784)$ FAR groups, and low $(\leq 5 \mathrm{ng} / \mathrm{ml})$ or high $(>5 \mathrm{ng} / \mathrm{ml})$ CEA groups. Furthermore, we evaluated the associations between these three indexes with clinicopathological features (Table 2). FPR and FAR were significantly associated with old age $(p<0.05)$, large tumor size $(p \leq 0.001)$, tumor invasion depth $(p<0.01)$, lymph node metastasis $(p<0.001)$, advanced TNM stage $(p<0.001)$, large Borrmann type $(p<0.01)$, and anemia status $(p \leq 0.001)$. Additionally, FAR was significantly related to high BMI $(p<0.05)$. However, FPR and FAR had no statistical correlations with gender, pathological differentiation, and CA199 levels $(p>0.05)$. CEA was significantly associated with tumor invasion depth $(p<0.001)$, advanced TNM stage $(p=0.003)$, and CA199 $(p=0.002)$. Nevertheless, CEA had no statistical correlations with gender, age, pathological differentiation, tumor size, lymph node metastasis, Borrmann type, BMI, and anemia status $(p>0.05)$. Mann-Whitney $U$ test indicated that the levels of FPR and FAR in patients with $\mathrm{T} 1+2$ depth, lymph nodes metastasis-negative, and stage I were lower than in those with $\mathrm{T} 3+4$ depth, lymph nodes metastasis-positive, and stage II/III, respectively $(p<0.05)$ (Additional file 4: Figure S2).

\section{Survival analysis and prognostic impact of FPR, FAR and FFC score}

In this study, Kaplan-Meier analysis was conducted to identify the prognostic significance of FPR, FAR, and FFC score. As revealed in Fig. 2, short OS was demonstrated to be significantly associated with high levels of FPR ( $\geq 0.0145$; Fig. 2a; $p<0.001)$, FAR ( $\geq 0.0784$; Fig. 2b; $p<0.001)$, CEA ( $>5 \mathrm{ng} / \mathrm{ml}$; Fig. $2 \mathrm{c} ; p=0.006)$, and FFC score ( $\geq 1$; Fig. 2 d; $p<0.001)$. Additionally, patients with $\mathrm{FFC}$ score $=3$ experienced the lowest survival rate compared with those belonging to the three other groups. Conversely, patients with FFC score $=0$ had the highest OS among the four groups. Kruskal-Wallis test indicated that there was no significant difference in terms of prediction of survival in the three different situations of FFC score $=2(p=0.812)$ (Additional file 5: Figure S3).

Cox proportional hazards model was selected for further survival analysis to evaluate the prediction models of baseline characteristics and preoperational FPR, FAR, and FFC score. As shown in Table 3, FPR $(\mathrm{HR}=2.499$, 95\% CI: $1.794-3.483, p<0.001)$, FAR (HR $=2.343,95 \%$ CI: $1.673-3.280, p<0.001)$, lymph node metastasis $(\mathrm{HR}=5.119,95 \%$ CI: 3.296-7.952, $p<0.001)$, TNM stage $(\mathrm{HR}=3.147,95 \% \mathrm{CI}: 2.385-4.151, p<0.001)$, Borrmann type $(\mathrm{HR}=1.433,95 \%$ CI: 1.238-1.658, $p<0.001)$, tumor size ( $\mathrm{HR}=3.587,95 \% \mathrm{CI}: 2.559-5.028$, $p<0.001)$, anemia status (HR $=1.512,95 \%$ CI: $1.071-$ 
Table 2 Correlation between the preoperative FPR and FAR with clinicopathological features in gastric cancer patients

\begin{tabular}{|c|c|c|c|c|c|c|c|c|c|}
\hline \multirow[t]{2}{*}{ Variables } & \multicolumn{2}{|l|}{ FPR } & \multirow[t]{2}{*}{$\boldsymbol{P}$ value } & \multicolumn{2}{|l|}{ FAR } & \multirow[t]{2}{*}{$\boldsymbol{P}$ value } & \multicolumn{2}{|l|}{ CEA } & \multirow[t]{2}{*}{$\boldsymbol{P}$ value } \\
\hline & $\begin{array}{l}<0.0145 \\
\mathrm{n}(\%)\end{array}$ & $\begin{array}{l}\geq 0.0145 \\
\text { n (\%) }\end{array}$ & & $\begin{array}{l}<0.0784 \\
\mathrm{n}(\%)\end{array}$ & $\begin{array}{l}\geq 0.0784 \\
\mathrm{n}(\%)\end{array}$ & & $\begin{array}{l}\leq 5 \mathrm{ng} / \mathrm{ml}, \\
\mathrm{n}(\%)\end{array}$ & $\begin{array}{l}>5 \mathrm{ng} / \mathrm{ml}, \\
\mathrm{n}(\%)\end{array}$ & \\
\hline Gender & & & 0.271 & & & 1.000 & & & 1.000 \\
\hline Male & $122(74.8)$ & $75(68.2)$ & & $106(72.1)$ & $91(72.2)$ & & $159(71.9)$ & $38(73.1)$ & \\
\hline Female & $41(25.2)$ & $35(31.8)$ & & $41(27.9)$ & $35(27.8)$ & & $62(28.1)$ & $14(26.9)$ & \\
\hline Age (years) & & & 0.013 & & & 0.002 & & & 0.277 \\
\hline$<60$ & $101(62.0)$ & $51(46.4)$ & & $95(64.6)$ & $57(45.2)$ & & $127(57.5)$ & $25(48.1)$ & \\
\hline$\geq 60$ & $62(38.0)$ & $59(53.6)$ & & $52(35.4)$ & $69(54.8)$ & & $94(42.5)$ & $27(51.9)$ & \\
\hline Pathological differentiation & & & 0.594 & & & 0.692 & & & 0.313 \\
\hline Moderate+Well & $51(31.3)$ & $31(28.2)$ & & $46(31.3)$ & $36(28.6)$ & & $63(28.5)$ & $19(36.5)$ & \\
\hline Poor & $112(68.7)$ & $79(71.8)$ & & $101(68.7)$ & $90(71.4)$ & & $158(71.5)$ & $33(63.5)$ & \\
\hline Tumor size $(\mathrm{cm})$ & & & 0.001 & & & $<0.001$ & & & 0.160 \\
\hline$<5$ & $110(67.5)$ & $51(46.4)$ & & $103(70.1)$ & $58(46.0)$ & & $135(61.1)$ & $26(50.0)$ & \\
\hline$\geq 5$ & $53(32.5)$ & $59(53.6)$ & & $44(29.9)$ & $68(54.0)$ & & $86(38.9)$ & $26(50.0)$ & \\
\hline Invasion depth & & & 0.006 & & & $<0.001$ & & & $<0.001$ \\
\hline $\mathrm{T} 1+\mathrm{T} 2$ & $64(39.3)$ & $25(22.8)$ & & $63(42.9)$ & $26(20.6)$ & & $82(37.1)$ & $7(13.5)$ & \\
\hline $\mathrm{T} 3+\mathrm{T} 4$ & $99(60.7)$ & $85(77.2)$ & & $84(57.1)$ & $100(79.4)$ & & $139(62.9)$ & $45(86.5)$ & \\
\hline Lymph nodes metastasis & & & $<0.001$ & & & $<0.001$ & & & 0.350 \\
\hline Negative & $81(49.7)$ & $30(27.3)$ & & $75(51.0)$ & $36(28.6)$ & & $93(42.1)$ & $18(34.6)$ & \\
\hline Positive & $82(50.3)$ & $80(72.7)$ & & $72(49.0)$ & $90(71.4)$ & & $128(57.9)$ & $34(65.4)$ & \\
\hline TNM stage & & & $<0.001$ & & & $<0.001$ & & & 0.003 \\
\hline । & $50(30.7)$ & $16(14.5)$ & & 49 (33.3) & $17(13.5)$ & & $62(28.1)$ & $4(7.7)$ & \\
\hline$\|$ & $53(32.5)$ & $25(22.7)$ & & $46(31.3)$ & $32(25.4)$ & & $56(25.3)$ & $22(42.3)$ & \\
\hline III & $60(36.8)$ & $69(62.8)$ & & $52(35.4)$ & $77(61.1)$ & & $103(46.6)$ & $26(50.0)$ & \\
\hline CA199 (U/ml) & & & 0.746 & & & 1.000 & & & 0.002 \\
\hline$\leq 37$ & $136(83.4)$ & $90(81.8)$ & & $122(83.0)$ & $104(82.5)$ & & $191(86.4)$ & $35(67.3)$ & \\
\hline$>37$ & $27(16.6)$ & $20(18.2)$ & & $25(17.0)$ & $22(17.5)$ & & $30(13.6)$ & $17(32.7)$ & \\
\hline Borrmann type & & & 0.009 & & & $<0.001$ & & & 0.149 \\
\hline 0 & $38(23.3)$ & $8(7.3)$ & & $38(25.9)$ & $8(6.3)$ & & $43(19.5)$ & $3(5.8)$ & \\
\hline I & $21(12.9)$ & $18(16.4)$ & & $17(11.6)$ & $22(17.5)$ & & $30(13.6)$ & $9(17.3)$ & \\
\hline$\|$ & $19(11.7)$ & $18(16.4)$ & & $12(8.1)$ & $25(19.8)$ & & $30(13.6)$ & $7(13.4)$ & \\
\hline III & $71(43.5)$ & $59(53.6)$ & & $68(46.3)$ & $62(49.2)$ & & $100(45.2)$ & $30(57.7)$ & \\
\hline IV & $14(8.6)$ & $7(6.3)$ & & $12(8.1)$ & $9(7.2)$ & & $18(8.1)$ & $3(5.8)$ & \\
\hline Body mass index $\left(\mathrm{kg} / \mathrm{m}^{2}\right)$ & & & 0.085 & & & 0.013 & & & 0.508 \\
\hline$<24$ & $105(64.4)$ & $82(74.5)$ & & $91(61.9)$ & $96(76.2)$ & & $149(67.4)$ & $38(73.1)$ & \\
\hline$\geq 24$ & $58(35.6)$ & $28(25.5)$ & & $56(38.1)$ & $30(23.8)$ & & $72(32.6)$ & $14(26.9)$ & \\
\hline Anemia status & & & 0.001 & & & $<0.001$ & & & 0.493 \\
\hline Negative & $130(79.8)$ & $66(60.0)$ & & $119(81.0)$ & $77(61.1)$ & & $161(72.9)$ & $35(67.3)$ & \\
\hline Positive & $33(20.2)$ & $44(40.0)$ & & $28(19.0)$ & 49 (38.9) & & $60(27.1)$ & $17(32.7)$ & \\
\hline
\end{tabular}

CEA carcinoembryonic antigen; CA199 carbohydrate antigen 199; FPR fibrinogen-to-prealbumin ratio; FAR fibrinogen-to-albumin ratio

2.134, $p=0.019)$, CEA $(\mathrm{HR}=1.686,95 \% \mathrm{CI}: 1.155-2.462$, $p=0.007)$, and CA199 (HR $=1.648,95 \% \mathrm{CI}: 1.106-2.455$, $p=0.014)$ were determined as statistically significant prognostic factors for OS by univariate analysis.
However, OS had no associations with age, gender, and pathological differentiation.

To further assess the independent prognostic predictor for OS, the factors with a $p$ value $<0.1$ in the univariate 

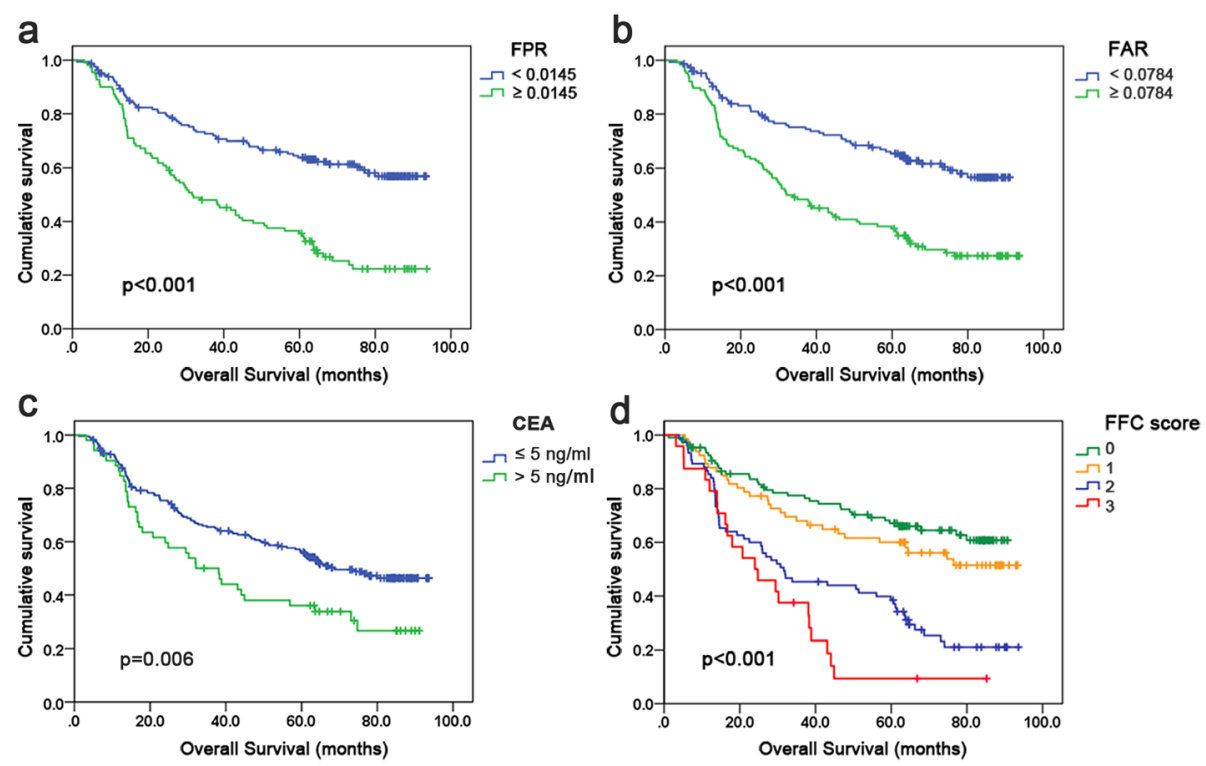

Fig. 2 Kaplan-Meier curves for OS according to the optimal cutoff value of FPR (a), FAR (b), CEA (c) and FFC score (d) in gastric cancer patients. Abbreviations: OS, overall survival; FPR, fibrinogen-to-prealbumin ratio; FAR, fibrinogen-to-albumin ratio; CEA, carcinoembryonic antigen; FFC, FPR-FAR-CEA

analysis were enrolled in the multivariate Cox proportional hazards model. Multivariate analysis showed FPR $(\mathrm{HR}=1.595,95 \%$ CI: $1.072-2.373, p=0.021)$, TNM stage $(\mathrm{HR}=3.564,95 \% \mathrm{CI}: 1.321-9.618, p=0.012)$, and tumor size $(\mathrm{HR}=1.958,95 \% \mathrm{CI}: 1.349-2.842, p<0.001)$ were significant independent predictors of OS. However, FAR, CEA, CA199, lymph node metastasis, Borrmann type, and anemia status had no significance in multivariate analysis.

The Cox proportional hazards model based on lymph node metastasis, TNM stage, Borrmann type, tumor size, anemia status and CA199 was used to further confirm the prognostic value of the FFC score. As shown in Table 3, univariate analysis showed that the FFC score is

Table 3 Univariate and multivariate analysis for OS in gastric cancer patients

\begin{tabular}{|c|c|c|c|c|}
\hline \multirow[t]{2}{*}{ Variables } & \multicolumn{2}{|l|}{ Univariate analysis } & \multicolumn{2}{|l|}{ Multivariate analysis } \\
\hline & $\mathrm{HR}(95 \% \mathrm{Cl})$ & $\boldsymbol{P}$ value & $\mathrm{HR}(95 \% \mathrm{Cl})$ & $\boldsymbol{P}$ value \\
\hline Gender & $0.742(0.505-1.091)$ & 0.129 & & \\
\hline Age & $1.270(0.916-1.761)$ & 0.152 & & \\
\hline Pathological differention & $1.191(0.909-1.562)$ & 0.205 & & \\
\hline Lymph nodes metastasis & 5.119 (3.296-7.952) & $<0.001$ & $1.646(0.864-3.135)$ & 0.130 \\
\hline TNM stage & $3.147(2.385-4.151)$ & $<0.001$ & 3.564 (1.321-9.618) & 0.012 \\
\hline Borrmann type & $1.433(1.238-1.658)$ & $<0.001$ & $0.980(0.808-1.187)$ & 0.834 \\
\hline Tumor size & 3.587 (2.559-5.028) & $<0.001$ & 1.958 (1.349-2.842) & $<0.001$ \\
\hline Body mass index & $0.884(0.619-1.264)$ & 0.500 & & \\
\hline Anemia status & $1.512(1.071-2.134)$ & 0.019 & $0.971(0.676-1.394)$ & 0.872 \\
\hline CEA & $1.686(1.155-2.462)$ & 0.007 & $1.422(0.959-2.107)$ & 0.080 \\
\hline CA199 & $1.648(1.106-2.455)$ & 0.014 & $1.043(0.689-1.579)$ & 0.842 \\
\hline FPR & $2.499(1.794-3.483)$ & $<0.001$ & 1.595 (1.072-2.373) & 0.021 \\
\hline FAR & $2.343(1.673-3.280)$ & $<0.001$ & $1.236(0.829-1.842)$ & 0.299 \\
\hline FFC score & 1.704 (1.446-2.009) & $<0.001$ & $1.414(1.189-1.683)$ & $<0.001$ \\
\hline
\end{tabular}



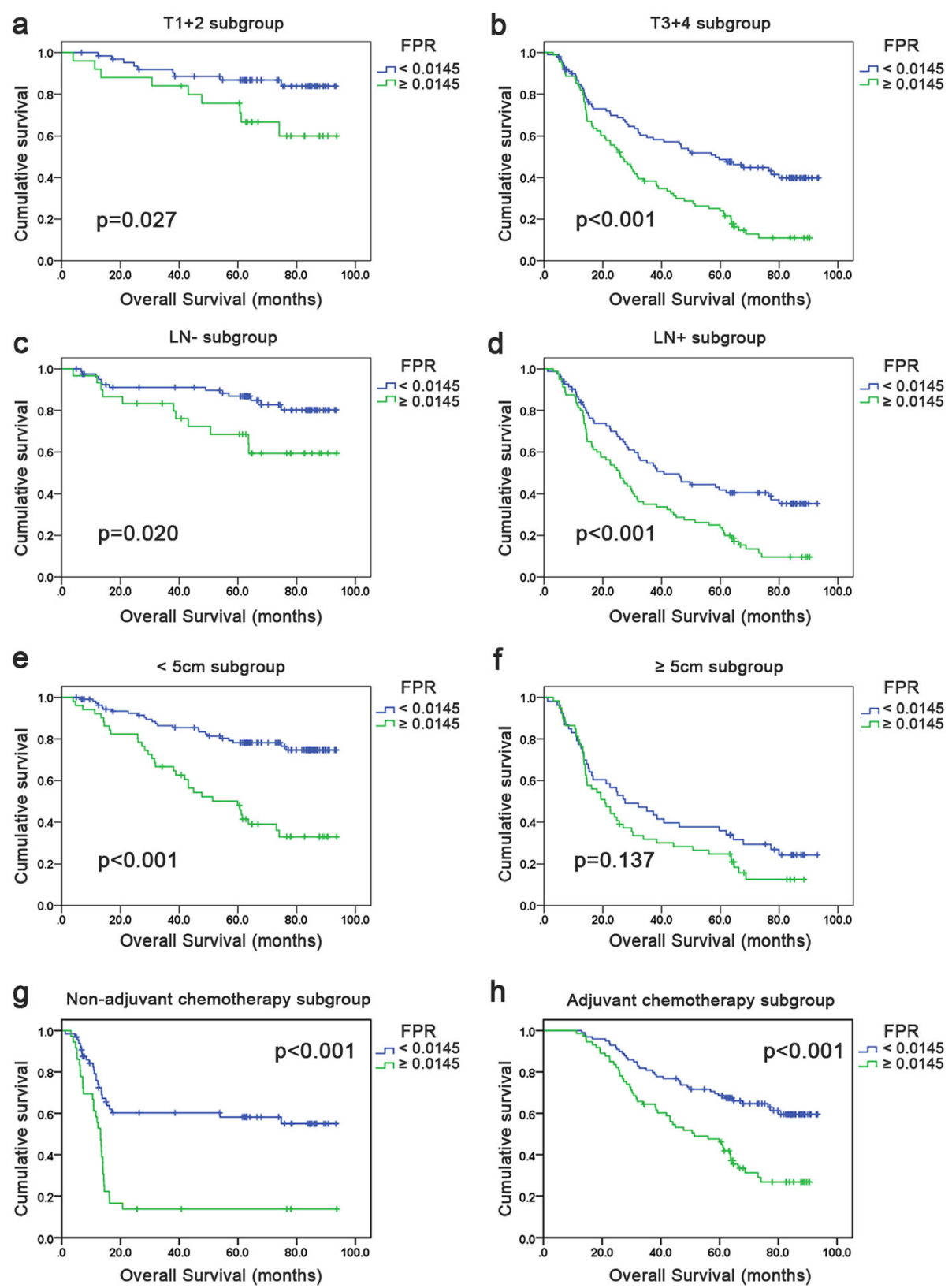

Fig. 3 Kaplan-Meier curves analyses for OS according to the optimal cutoff value of FPR in each subgroup. a T1 + 2 subgroup; b T3+ 4 subgroup; c LN- subgroup; $\mathbf{d} L N+$ subgroup; e tumor size $<5 \mathrm{~cm}$ subgroup; $\mathbf{f}$ tumor size $\geq 5 \mathrm{~cm}$ subgroup; $\mathbf{g}$ non-adjuvant chemotherapy subgroup; $\mathbf{h}$ adjuvant chemotherapy subgroup. Abbreviations: OS, overall survival; FPR, fibrinogen-to-prealbumin ratio; LN-, lymph nodes metastasis-negative; LN+, lymph nodes metastasis-positive

a significant prognostic factor $(\mathrm{HR}=1.704,95 \% \mathrm{CI}$ : 1.446-2.009, $p<0.001)$. The FFC score was also a significant independent predictor in multivariate analysis $(\mathrm{HR}=1.414,95 \% \mathrm{CI}: 1.189-1.683, p<0.001)$.

\section{Subgroup analysis}

Individual subgroup analyses were performed based on tumor invasion depth, lymph node metastasis, adjuvant chemotherapy, and tumor size to investigate the prognostic significance of FPR, and FFC score in patients with GC. As shown in Fig. 3, extended OS was found in patients with low FPR in the $\mathrm{T} 1+2$ and $\mathrm{T} 3+4$ subgroups $(p=0.027$ and $p<0.001$, respectively), lymph nodes metastasis-negative and -positive subgroups ( $p=$ 0.020 and $p<0.001$, respectively), non-adjuvant chemotherapy and adjuvant chemotherapy subgroups $(p<0.001)$, and $<5 \mathrm{~cm}$ subgroup $(p<0.001)$, but not in the $\geq 5 \mathrm{~cm}$ subgroup $(p=0.137)$. In addition, short OS 
was found in patients with high FFC score $(\geq 2)$ in the $\mathrm{T} 1+2$ and $\mathrm{T} 3+4$ subgroups $(p=0.021$ and $p<0.001$, respectively), lymph nodes metastasis-negative and -positive subgroups $(p<0.001)$, non-adjuvant chemotherapy and adjuvant chemotherapy subgroups $(p<0.001)$, and $<5 \mathrm{~cm}$ subgroup $(p<0.001)$, but not in the $\geq 5 \mathrm{~cm}$ subgroup $(p=0.093)$ (Additional file 6: Figure S4). The subgroup analyses based on TNM for FPR, FAR and FFC score were also performed in our study. Short OS was found in high FPR, FAR and FFC score $(\geq$ $2)$ in both stage I and III subgroup $(p<0.05)$. While, there were no statistically significant difference in OS in high or low FPR, FAR and FFC score in the stage II subgroup $(p>0.05)$ (Additional file 7: Figure S5). In this study, we also evaluated FFC score in high FPR group $(\geq$ 0.0145 ), and we found the OS in patients with high FFC score was shorter $(p=0.019)$ (Additional file 8: Figure S6).

\section{Discussion}

The association between GC and chronic inflammation has attracted extensive attention over the past decades. Currently, chronic inflammation has been recognized as a hallmark of GC and can occur in GC initiation and progression [11]. For instance, Helicobacter pylori infection triggers gastric carcinogenesis with a unique and complex process involving chronic atrophic gastritis, intestinal metaplasia, and occurrence of invasive carcinoma [12, 13]. Emerging evidence indicated that immune cells and inflammatory cytokines play vital roles in the progression of GC by regulating the tumor microenvironment $[14,15]$. Furthermore, understanding inflammatory mechanisms in GC will establish personalized immune-related treatment against cancer and identify novel diagnostic and prognostic biomarkers for patients with GC [16]. Consequently, chronic inflammation plays a key role in $\mathrm{GC}$, and inflammation-associated factors provide a rich resource for biomarkers.

In recent years, numerous studies described the essential role of inflammation-related markers in the diagnosis and prognosis for GC. For example, elevated NLR and PLR and decreased LMR in patients with both early and advanced GC are related to poor prognosis $[4,5,17$, 18]. Similarly, patients with GC and high AGR present poor survival compared with those with low AGR [19]. Many inflammation-related markers have been explored as a prognostic predictor for GC; however, additional reliable indices are still required.

Fibrinogen is a pivotal coagulation-related protein in mediating communication between hemostatic components and cancer biology. Tumor progression and metastasis can be promoted by fibrinogen through different mechanisms, such as stimulation of angiogenesis, promotion of platelet adhesion, and enhancement of tumor cell proliferation and migration by binding to growth factors [20]. In addition, patients with GC and hyperfibrinogenemia have increased risk of poor clinical outcome and lymphatic metastasis, and treatment for such patients can be optimized by evaluating peripheral fibrinogen. For instance, in a large cohort of 1196 patients with GC, elevated fibrinogen was found to be positively correlated with low survival [21]. Similarly, in 1090 patients with GC who underwent surgery, preoperative fibrinogen was proposed as an independent prognostic biomarker [22]. Furthermore, circulating albumin and prealbumin are indicators for assessing nutritional status and markers of immune status. Tumor progression can be restrained by albumin by stabilizing DNA replication and enhancing immunity response [23]. Albumin is widely used as an ideal drug delivery platform in anti-inflammatory and anticancer therapy because it accumulates at inflammation and tumor sites $[24,25]$. Additionally, hypoalbuminemia has been associated with poor prognosis in GC due to malnutrition and postoperative complications [26].

Fibrinogen, albumin, and prealbumin are important components of inflammation-associated GC. However, the prognostic value of their combination in GC remains unclear. In this study, we demonstrated that both preoperative FPR and FAR were promising noninvasive prognostic biomarkers for GC, and FPR was an independent prognostic predictor for resectable GC. These results were consistent with previous research on CRC $[10,27]$. Increasing studies have used FAR as an independent prognostic factor in patients with resectable ESCC, hepatocellular carcinoma, and advanced nonsmall cell lung cancer, and high levels of FAR can lead to high risk of recurrence and unfavorable OS $[8,28$, 29]. Unfortunately, FAR was not an independent prognostic predictor for resectable GC in our study. Our results suggested that FPR is a more suitable prognostic biomarker for GC than FAR.

In subgroup analysis, we found that short OS was related to high FPR and FFC score in patients with GC across all depths, lymph nodes status, and AJCC stage subgroups. For patients with a tumor size of $\geq 5 \mathrm{~cm}, \mathrm{FPR}$ and FFC score were not related to OS. These findings suggested that both FPR and FFC score were highly useful predictors for small tumors in GC.

Similar to a single inflammatory biomarker, emerging prognostic scores based on immune and nutritional status are attracting attention as novel and superior predictors of prognosis in GC. For instance, GPS or modified GPS based on cancer-related inflammation is an independent predictor of GC survival and can be used as a predictor for patients with GC undergoing platinumbased chemotherapy [30, 31]. Moreover, low prognostic nutritional index (PNI), which is calculated from the 
combination of BMI, lymphocyte count, and albumin, is associated with poor clinical outcome in resectable GC and can act as an independent risk factor [32]. Similarly, controlling nutritional status (CONUT) score, which is obtained from total lymphocyte count, total cholesterol level, and albumin, is associated with survival in patients with GC undergoing curative gastrectomy [33]. Despite extensive work on prognostic scores, additional effective and reliable prognostic scores need to be investigated. In this study, we demonstrated that FFC score was superior to FAR or CEA, and considered an independent prognostic factor for GC. In addition, short OS was found in patients with high FFC score in high FPR group ( $p=$ 0.019). It indicated that FFC score could be used for further stratification for high FPR group. Compared to purely using FPR, FFC score could provide more precise prognostic information for the patients with high FPR.

In recent years, with the substantial progress and clinical application of artificial intelligence, machine learning along with the explosive growth of biomedical big-data has generated much interest in developing clinical informatics tools for disease diagnosis, staging, and prognosis [34]. There was a strong power in machine learning approach on constructing new scoring system based on existing clinical indicators. Despite some scoring systems for cancer have been created and validated by the novel method of machine learning [35], the evidence of scoring system based on inflammation biomarkers for gastric cancer is still lacking and elusive. Thus, more studies using the novel method of machine learning are needed to discover more effective biomarker in gastric cancer.

This study was the first to establish FFC score, a novel scoring system based on FPR, FAR, and CEA, and investigate its prognostic value in resectable GC. However, our study still had several potential limitations. First, cases enrolled in the retrospective study were from a single center, so sample size was limited and had selection bias. Second, diverse adjuvant chemotherapy may lead to heterogeneous clinical outcomes. Third, the consistency of peripheral blood results with tumor tissue still need further investigation. Fourth, although the optimal cutoff values was determined by ROC curves, the sensitivity of the optimal cutoff values for FPR and FAR is limited. This may due to the highly heterogeneous of gastric cancer, and a relatively small size of enrolled patients. Therefore, further research with a large cohort from multiple centers are needed to confirm the cutoff values for FPR and FAR, and validate the prognostic roles of FPR level and FFC score in GC.

\section{Conclusions}

Preoperative FPR and FFC score, which are convenient, quick, cost-effective, and entail minimal pain, could be used as prospective noninvasive prognostic biomarkers for resectable GC. Low FPR and FFC score may improve the survival of patients with resectable GC.

\section{Supplementary information}

Supplementary information accompanies this paper at https://doi.org/10. 1186/s12885-020-06866-6.

Additional file $\mathbf{1}$ Table S1. Baseline characteristics of gastric cancer patients.

Additional file 2 Table S2. ROC curve analyses and optimal cutoff values of FPR and FAR for 5-year OS in gastric cancer.

Additional file 3 Figure S1. ROC curve analyses and permutation test of FFC score. (a) The AUC of FFC score reached 0.710 by using the median value of 1.5 as the cutoff value. (b) A 200 times permutation test for (a) showed that FFC score could be significantly distinguished from random effect.

Additional file 4 Figure S2. The correlation of preoperative FPR and FAR with pathological features. (a) FPR with invation depth; (b) FPR with lymph node statu; (c) FPR with pathological tumor stage; (d) FAR with invation depth; (e) FAR with lymph node statu; (f) FAR with pathological tumor stage. The comparisons between two groups were assessed using Mann-Whitney $U$ test.

Additional file 5 Figure S3. Differences in overall survival in the different situations of FFC score $=2$ (Kruskal-Wallis test, $p=0.812$ ).

Additional file 6 Figure S4. Kaplan-Meier curves analyses for OS according to FFC score in each subgroup. (a) T1 +2 subgroup; (b) T3 + 4 subgroup; (c) LN- subgroup; (d) LN+ subgroup; (e) tumor size $<5 \mathrm{~cm}$ subgroup; ( $f$ ) tumor size $\geq 5 \mathrm{~cm}$ subgroup; (g) non-adjuvant chemotherapy subgroup; (h) adjuvant chemotherapy subgroup.

Additional file 7 Figure S5. Kaplan-Meier curves analyses for OS according to the optimal cutoff value of FPR, FAR, and FFC score in TNM subgroup. (a) FPR-stage I subgroup; (b) FPR-stage II subgroup; (c) FPRstage III subgroup; (d) FAR-stage I subgroup; (e) FAR-stage II subgroup; (f) FAR-stage III subgroup; (g) FFC score-stage I subgroup; (h) FFC scorestage II subgroup; (i) FFC score-stage III subgroup.

Additional file 8 Figure S6. Kaplan-Meier curves analyses for OS according to FFC score in high FPR group $(\geq 0.0145)$

\section{Abbreviations}

AUC: Area under the receiver operating characteristic curve; BMI: Body mass index; CEA: Carcinoembryonic antigen; CA199: Carbohydrate antigen 199;

$\mathrm{Cl}$ : Confidence interval; CONUT: Controlling nutritional status; CRC: Colorectal cancer; ESCC: Esophageal squamous cell carcinoma; FAR: Fibrinogen-toalbumin ratio; FPR: Fibrinogen-to-prealbumin ratio; FFC: FPR-FAR-CEA; GC: gastric cancer; GPS: Glasgow prognostic score; LMR: Lymphocyte-tomonocyte ratio; NLR: Neutrophil-to-lymphocyte ratio; OS: Overall survival; PLR: Platelet-to-lymphocyte ratio; PNI: Prognostic nutritional index; ROC: Receiver operating characteristic.

\section{Acknowledgements}

Not applicable.

\section{Authors' contributions}

ST, YZ and YQZ conceived and designed the study. LL, JC and JZ collected the clinical information. ST, QX and JS performed statistical analysis and wrote the manuscript. YZ and YQZ reviewed and edited the manuscript. All the authors read and approved the final manuscript.

\section{Funding}

This work was supported by the National Natural Science Foundation of China (grant numbers 81872427, 81672428), the Haiyan Fund Project of Harbin Medical University Cancer Hospital (grant numbers JJQN2019-02, JJZD2020-17), and the Health Commission of Heilongjiang (grant number 2019-060). The funding body had no involvement in the design of the 
study, collection, analysis, and interpretation of data and in writing the manuscript.

\section{Availability of data and materials}

The data are available from the corresponding author on reasonable request.

\section{Ethics approval and consent to participate}

All participants gave written informed consent. This study was conducted according to the Declaration of Helsinki and was approved by the Institutional Ethics Committee of Harbin Medical University Cancer Hospital.

\section{Consent for publication}

Not applicable.

\section{Competing interests}

The authors declare that they have no competing interests.

Received: 12 November 2019 Accepted: 14 April 2020

Published online: 06 May 2020

\section{References}

1. Bray F, Ferlay J, Soerjomataram I, Siegel RL, Torre LA, Jemal A. Global cancer statistics 2018: GLOBOCAN estimates of incidence and mortality worldwide for 36 cancers in 185 countries. CA Cancer J Clin. 2018;68(6):394-424.

2. Chen W, Sun K, Zheng R, Zeng H, Zhang S, Xia C, et al. Cancer incidence and mortality in China, 2014. Chin J Cancer Res. 2018;30(1):1-12.

3. Karimi P, Islami F, Anandasabapathy S, Freedman ND, Kamangar F. Gastric cancer: descriptive epidemiology, risk factors, screening, and prevention. Cancer Epidemiol Biomark Prev. 2014;23(5):700-13.

4. Ocana A, Nieto-Jimenez C, Pandiella A, Templeton AJ. Neutrophils in cancer: prognostic role and therapeutic strategies. Mol Cancer. 2017;16(1):137.

5. Deng Q, He B, Liu X, Yue J, Ying H, Pan Y, et al. Prognostic value of preoperative inflammatory response biomarkers in gastric cancer patients and the construction of a predictive model. J Transl Med. 2015:13:66.

6. Jiang X, Hiki N, Nunobe S, Kumagai K, Kubota T, Aikou S, et al. Prognostic importance of the inflammation-based Glasgow prognostic score in patients with gastric cancer. Br J Cancer. 2012;107(2):275-9.

7. Lin JX, Lin JP, Xie JW, Wang JB, Lu J, Chen QY, et al. Prognostic importance of the preoperative modified systemic inflammation score for patients with gastric cancer. Gastric Cancer. 2019;22(2):403-12.

8. Ying J, Zhou D, Gu T, Huang J, Liu H. Pretreatment albumin/fibrinogen ratio as a promising predictor for the survival of advanced non small-cell lung cancer patients undergoing first-line platinum-based chemotherapy. BMC Cancer. 2019;19(1):288

9. Liang Y, Wang W, Que Y, Guan Y, Xiao W, Fang C, et al. Prognostic value of the fibrinogen/albumin ratio (FAR) in patients with operable soft tissue sarcoma. BMC Cancer. 2018;18(1):942.

10. Sun F, Peng HX, Gao QF, Li SQ, Zhang J, Chen QG, et al. Preoperative circulating FPR and CCF score are promising biomarkers for predicting clinical outcome of stage II-III colorectal cancer patients. Cancer Manag Res. 2018;10:2151-61.

11. Hunt RH, Camilleri M, Crowe SE, El-Omar EM, Fox JG, Kuipers EJ, et al. The stomach in health and disease. Gut. 2015;64(10):1650-68.

12. Yoshida T, Kato J, Inoue I, Yoshimura N, Deguchi H, Mukoubayashi $\mathrm{C}$, et al. Cancer development based on chronic active gastritis and resulting gastric atrophy as assessed by serum levels of pepsinogen and helicobacter pylori antibody titer. Int J Cancer. 2014;134(6):1445-57.

13. Wessler S, Krisch LM, Elmer DP, Aberger F. From inflammation to gastric cancer - the importance of hedgehog/GLI signaling in helicobacter pyloriinduced chronic inflammatory and neoplastic diseases. Cell Commun Signal. 2017;15(1):15

14. Echizen $\mathrm{K}$, Hirose $\mathrm{O}$, Maeda $\mathrm{Y}$, Oshima M. Inflammation in gastric cancer: interplay of the COX-2/prostaglandin E2 and toll-like receptor/MyD88 pathways. Cancer Sci. 2016;107(4):391-7

15. Li TJ, Jiang YM, Hu YF, Huang L, Yu J, Zhao LY, et al. Interleukin-17producing neutrophils link inflammatory stimuli to disease progression by promoting angiogenesis in gastric Cancer. Clin Cancer Res. 2017;23(6):157585.

16. McLean MH, El-Omar EM. Genetics of gastric cancer. Nat Rev Gastroenterol Hepatol. 2014;11(11):664-74.
17. Ock CY, Nam AR, Lee J, Bang JH, Lee KH, Han SW, et al. Prognostic implication of antitumor immunity measured by the neutrophil-lymphocyte ratio and serum cytokines and angiogenic factors in gastric cancer. Gastric Cancer. 2017:20(2):254-62.

18. Lian L, Xia YY, Zhou C, Shen XM, Li XL, Han SG, et al. Application of platelet/ lymphocyte and neutrophil/lymphocyte ratios in early diagnosis and prognostic prediction in patients with resectable gastric cancer. Cancer Biomark. 2015;15(6):899-907.

19. Liu J, Chen S, Geng Q, Liu X, Kong P, Zhan Y, et al. Prognostic value of pretreatment albumin-globulin ratio in predicting long-term mortality in gastric cancer patients who underwent D2 resection. Onco Targets Ther 2017;10:2155-62.

20. Repetto O, De Re V. Coagulation and fibrinolysis in gastric cancer. Ann N Y Acad Sci. 2017;1404(1):27-48.

21. Yu X, Hu F, Yao Q, Li C, Zhang H, Xue Y. Serum fibrinogen levels are positively correlated with advanced tumor stage and poor survival in patients with gastric cancer undergoing gastrectomy: a large cohort retrospective study. BMC Cancer. 2016;16:480.

22. Yu W, Wang Y, Shen B. An elevated preoperative plasma fibrinogen level is associated with poor overall survival in Chinese gastric cancer patients. Cancer Epidemiol. 2016;42:39-45

23. Seaton K. Albumin concentration controls cancer. J Natl Med Assoc. 2001; 93(12):490-3.

24. Sleep D. Albumin and its application in drug delivery. Expert Opin Drug Deliv. 2015;12(5):793-812.

25. Bern M, Sand KM, Nilsen J, Sandlie I, Andersen JT. The role of albumin receptors in regulation of albumin homeostasis: implications for drug delivery. J Control Release. 2015;211:144-62.

26. Pacelli F, Bossola M, Rosa F, Tortorelli AP, Papa V, Doglietto GB. Is malnutrition still a risk factor of postoperative complications in gastric cancer surgery? Clin Nutr. 2008;27(3):398-407.

27. Chen QG, Zhang L, Sun F, Li SQ, You XH, Jiang YH, et al. Elevated FPR confers to radiochemoresistance and predicts clinical efficacy and outcome of metastatic colorectal cancer patients. Aging (Albany NY). 2019;11(6): 1716-32.

28. Xu Q, Yan Y, Gu S, Mao K, Zhang J, Huang P, et al. A novel inflammationbased prognostic score: the fibrinogen/albumin ratio predicts prognoses of patients after curative resection for hepatocellular carcinoma. J Immunol Res. 2018;2018:4925498.

29. Tan Z, Zhang M, Han Q, Wen J, Luo K, Lin P, et al. A novel blood tool of cancer prognosis in esophageal squamous cell carcinoma: the fibrinogen/ albumin ratio. J Cancer. 2017:8(6):1025-9.

30. Wen J, Bedford M, Begum R, Mitchell H, Hodson J, Whiting J, et al. The value of inflammation based prognostic scores in patients undergoing surgical resection for oesophageal and gastric carcinoma. J Surg Oncol. 2018;117(8):1697-707.

31. Dupre A, Malik HZ. Inflammation and cancer: what a surgical oncologist should know. Eur J Surg Oncol. 2018:44(5):566-70.

32. Hirahara N, Tajima Y, Fujii Y, Kaji S, Yamamoto T, Hyakudomi R, et al. Prognostic nutritional index as a predictor of survival in resectable gastric cancer patients with normal preoperative serum carcinoembryonic antigen levels: a propensity score matching analysis. BMC Cancer. 2018;18(1):285.

33. Kuroda D, Sawayama H, Kurashige J, Iwatsuki M, Eto T, Tokunaga R, et al. Controlling nutritional status (CONUT) score is a prognostic marker for gastric cancer patients after curative resection. Gastric Cancer. 2018;21(2): 204-12.

34. Le Berre C, Sandborn WJ, Aridhi S, Devignes MD, Fournier L, Smail-Tabbone $M$, et al. Application of artificial intelligence to gastroenterology and Hepatology. Gastroenterology. 2020;158(1):76-94 e72.

35. Dong TS, Kalani A, Aby ES, Le L, Luu K, Hauer M, et al. Machine learningbased development and validation of a scoring system for screening highrisk esophageal Varices. Clin Gastroenterol Hepatol. 2019;17(9):1894-901 e1891.

\section{Publisher's Note}

Springer Nature remains neutral with regard to jurisdictional claims in published maps and institutional affiliations. 